\title{
Ocular Ischemic Syndrome as a Clue for Carotid Artery Stenosis
}

\author{
Nazanin Ebrahimiadib ${ }^{1}$, Maziyar Irannejad ${ }^{1}$, Arash Alizadeh ${ }^{1}$, Elias Khalili Pour ${ }^{1}$ and Ehsan Khalilipur ${ }^{2 *}$ \\ ${ }^{1}$ Retina ward, Farabi eye hospital, Tehran university of medical scences, Tehran, IRAN \\ ${ }^{2}$ Cardiovascular intervention research center, Rajaie cardiovascular medical and research center, Iran university of medical scences, Tehran, Iran
}

Submission: May 07, 2021; Published: May 26, 2021

*Corresponding author: Ehsan Khalilipur, Cardiovascular intervention research center, Rajaie cardiovascular medical and research center, Iran university of medical scences, Tehran, Iran

Abstract

In current medical practice, all symptoms of a patient could reveal a systemic involvement of an inflammatory condition which primary presentation might not be vivid enough for diagnosis of the original disease. In this case report, a painful eye of a patient lead us to diagnose stenosis of carotid artery and endovascular therapy consequently subsided patient visual acuity and prevented future devastating results.

\section{Case Presentation}

A 65-year-old gentleman with no prior history of diabetes and cardiovascular disease presented with periorbital pain and gradual decreased vision in the left eye from 6 months ago. Due to ocular angina, the patient was treated with topical steroids and non-steroidal anti-inflammatory drugs (NSAIDs) for more than 5 months. The best-corrected visual acuity of the left eye was 1/10. Fundus examination revealed increased tessellation and scattered blot hemorrhages around the arcades and midperipheral region that were not accompanied by venous tortuosity and dilatation (Figure 1A, B). Anterior segment examination revealed cellular reaction, faint and fluffy neovascularization of the iris (NVI) that could be easily missed in cursory examination (Figure 1C). Optical coherence tomography (OCT) revealed atrophic and disorganized inner retinal layers and optical coherence tomography angiography (OCTA) showed noticeable decreased vascular density in both superficial and deep capillary plexi (not shown in Figure 1). Fluorescein angiography (FA) exhibited left side choroidal filling delay and frosted branch angiitis and vasculitis-like appearance compared to the right side (Figure $1 \mathrm{D}-\mathrm{G}$ ), and diagnosis of Ocular Ischemic Syndrome (OIS) was suspected. Carotid arteries computed tomography angiography confirmed stenosis of the left side common carotid and subclavian artery (Figure $1 \mathrm{H}$ ) so the patient was referred to the cardiologist for interventional cardiologist for carotid artery endovascular therapy.
At the angiography of aortic arc, there was a significant stenosis of left carotid artery but the stenosis of left subclavian was moderate and as the patient had no symptom relevant to subclavian artery, we decided to just intervene on left carotid artery. We performed the procedure from right femoral access and with use of embolic protection device and atropine before stent deployment, we used Carotid Wallstent 40 (Boston Scientific Corporation) and the procedure was terminated without any central or peripheral complications and 2 months follow-up visit of patient in cardiology and ophthalmology clinic revealed no complaint and he had a visual acuity of 8/10.

\section{Discussion}

As the mortality rate of patients with OIS over 5 years is about $40 \%$, ophthalmologists need to timely recognize the manifestations and symptoms of patients with OIS [1]. Since OIS is a rare condition and its symptoms overlap with other eye conditions, some cases are misdiagnosed, similar to this case in that ocular manifestations including orbital pain, were attributed to the uveitis for more than 5 months. Pain in the influenced eye or periorbital region is present in about $40 \%$ of cases and is referred to as "ocular angina." [2]. Patchy and delayed choroidal filling in FA is seen in $60 \%$ of eyes with the OIS. The filling may be delayed by a minute or more in some cases [2]. Late arterial staining may imitate frosted branch angiitis on angiography and arise due to 


\section{Journal of Cardiology \& Cardiovascular Therapy}

injury to endothelium owing to ischemia and can be found in up to $85 \%$ of cases [3]. Increased fundus tessellation in the affected eye in comparison to the contralateral eye as a useful clue can be attributed to atrophy of retinal layers over the choroidal vessels. As in our patient, referral of patient after diagnosis to a cardiologist and neurologist is mandatory and the diagnosis is a clue to assess other vascular bed specifically ipsilateral carotid artery vasculature [4].

In conclusion, precise diagnosis of patients with OIS can easily be missed. Hence, careful and meticulous ophthalmic examination, carotid examination of patients with ocular angina and comparing the manifestations of the affected eye with the contralateral eye can be very supportive and can save a patient's life and sight.

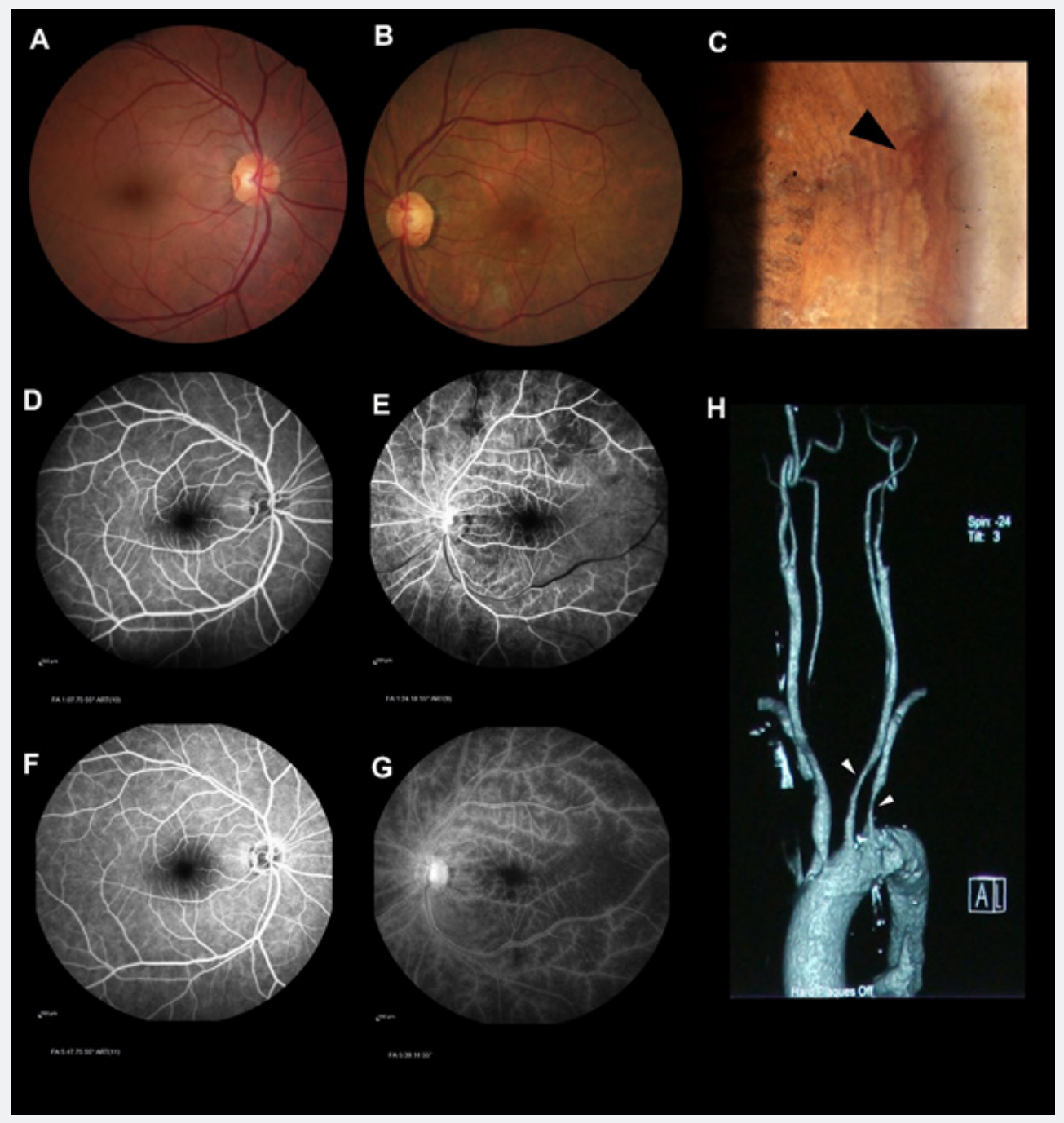

Figure 1: A, Colour fundus photo of the right eye shows normal appearance. B, Colour fundus photo of the left eye shows increased tessellation and scattered blot hemorrhages around the arcades and midperipheral region that are not accompanied by venous tortuosity and dilatation. $\mathrm{C}$, Neovascularization of the iris (NVI) in the left eye (black arrowhead). Fluorescein angiography of the right eye in the early $(D)$ and late $(F)$ phases corresponding to the left eye $(E, G)$ shows left side choroidal filling delay and frosted branch angiitis, and vasculitis-like appearance. Carotid arteries computed tomography angiography confirmed stenosis of the left side common carotid artery and subclavian artery (white arrowhead)

\section{Conflict of Interest}

No potential conflict of interest relevant to this article was reported.

\section{References}

1. Sivalingam A, Brown GC, Magargal LE, Menduke H (1989) The ocular ischemic syndrome. II. Mortality and systemic morbidity. Int Ophthalmol 13(3): 187-191. 
2. Brown GC, Magargal LE (1988) The ocular ischemic syndrome Clinical, fluorescein angiographic and carotid angiographic features. Int Ophthalmol 11(4): 239-251.

3. Sood G, Siddik AB (2021) Ocular Ischemic Syndrome. StatPearls Publishing.
4. Dzierwa K, Pieniazek P, Musialek P, Piatek J, Tekieli L, et al (2011) Treatment startegies in severe symptomatic carotid and coronary artery disease. Med Sci Monit 17(8): RA191-197.

Your next submission with Juniper Publishers
will reach you the below assets
- Quality Editorial service
- Swift Peer Review
- Reprints availability
- E-prints Service
- Manuscript Podcast for convenient understanding
- Global attainment for your research
- Manuscript accessibility in different formats
( Pdf, E-pub, Full Text, Audio)
- Unceasing customer service
Track the below URL for one-step submission
https://juniperpublishers.com/online-submission.php

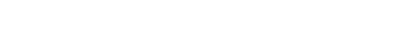

